Journal of Computational Neuroscience 15, 335-345, 2003 (C) 2003 Kluwer Academic Publishers. Manufactured in The Netherlands.

\title{
Linear and Nonlinear Measures and Seizure Anticipation in Temporal Lobe Epilepsy
}

\author{
DINGZHOU LI \\ Physics Department, University of Michigan, Ann Arbor, MI, USA \\ WEIPING ZHOU \\ Department of Biostatistics, University of Michigan, Ann Arbor, MI, USA \\ IVO DRURY \\ Department of Neurology, Henry Ford Health System, Detroit, MI, USA
}

\section{ROBERT SAVIT}

Michigan Center for Theoretical Physics, University of Michigan, Ann Arbor, MI, USA; Physics Department, University of Michigan, Ann Arbor, MI, USA; Center for the Study of Complex Systems and Biophysics Research Division, University of Michigan, Ann Arbor, MI, USA

savit@umich.edu

Received January 13, 2003; Revised June 27, 2003; Accepted July 9, 2003

\author{
Action Editor: E. Bard Ermentrout
}

\begin{abstract}
In a recent paper, we showed that the value of a nonlinear quantity computed from scalp electrode data was correlated with the time to a seizure in patients with temporal lobe epilepsy. In this paper we study the relationship between the linear and nonlinear content and analyses of the scalp data. We do this in two ways. First, using surrogate data methods, we show that there is important nonlinear structure in the scalp electrode data to which our methods are sensitive. Second, we study the behavior of some simple linear metrics on the same set of scalp data to see whether the nonlinear metrics contain additional information not carried by the linear measures. We find that, while the nonlinear measures are correlated with time to seizure, the linear measures are not, over the time scales we have defined. The linear and nonlinear measures are themselves apparently linearly correlated, but that correlation can be ascribed to the influence of a small set of outliers, associated with muscle artifact. A remaining, more subtle relation between the variance of the values of a nonlinear measure and the expectation value of a linear measure persists. Implications of our observations are discussed.
\end{abstract}

Keywords: seizure anticipation, temporal lobe epilepsy

\section{Introduction}

The problem of seizure anticipation in patients with epilepsy has attracted significant attention in the past few years. Success in developing a reliable ambula- tory method of seizure anticipation would have important implications both for the quality of life of people with epilepsy, and for the development of short term strategies to either limit or abort an impending seizure. Recently, we showed that a certain nonlinear measure 
computed using data from scalp electrodes is positively correlated with the time to a seizure in a cohort of patients with mesiobasal temporal lobe epilepsy (Li et al., 2003a, 2003b). These results suggest that this measure can be used as the basis for an ambulatory method of seizure anticipation. In this paper we study the relationship between time to seizure and two simple linear measures computed for the same set of data. By "linear" with reference to a time series, we mean all the information from the time series that is encoded in one of the following (equivalent) sets of information:

(i) The coefficients of a linear auto regressive fit to the data.

(ii) The power spectrum.

(iii) The values of all the linear two-point correlation functions.

Any other information, and specifically, information that involves the relative phases of the Fourier coefficients (as opposed to just their amplitudes) constitutes nonlinear information about the time series.

There are a number of reasons spanning a range of theoretical, practical and biological considerations to study the relationship between linear and nonlinear information in the context of seizure anticipation. First, questions have been raised by several different groups concerning the value-added utility of nonlinear analyses for seizure anticipation. See, for example, the older works by Theiler (1995), Rapp (1992), Pritchard and Duke (1992), and Paulus (1993) among others. More recently, a comparative study of the efficacy of linear versus nonlinear techniques yielded the conclusion that nonlinear techniques added little to seizure anticipation (Jerger et al., 2001). Finally, McSharry et al. (2003) argued that the change in behavior of a nonlinear measure used by Martiniere et al. (1998) as a seizure approached could be explained by the changes in the standard deviation of the time series, a purely linear measure. From a practical point of view, there is also good reason to address this issue. In general, nonlinear measures are more computationally intensive to estimate than are linear ones. If one seeks to develop a reliable ambulatory method of seizure anticipation, one would want to use the simplest possible metric that will do the job. It is important, therefore, to determine whether, in practice, nonlinear metrics have anything to offer over linear ones. Finally, there may be dynamical, biological implications if nonlinear measures add information over that contained in linear measures. For all these reasons, it is useful to compare the performance of some simple linear measures with our nonlinear measures. In addition to comparing the behavior of linear and nonlinear metrics on our data set, it is of interest to independently establish the extent to which there is nonlinear structure in the data set to which our nonlinear metrics are sensitive. In this paper we address the second issue by using the method of surrogate data, and we show that our nonlinear metrics behave very differently on linear surrogate data sets than they do on the real scalp data. Returning to our real scalp data, we show that, unlike our nonlinear measures described in earlier work, a set of very simple linear measures are not strongly correlated with time to seizure, over the time scales in which we are interested. On the other hand, there is, apparently, a functional relationship between the linear and nonlinear measures.

The rest of this paper is organized as follows: In the next section we provide a brief review of our nonlinear measures and summary of their behavior on the set of scalp data we have analyzed. In Section 3 we first describe the results of our surrogate data analysis which establishes the presence of strictly non-linear effects to which our metrics are sensitive. We then introduce some simple linear measures and describe their behavior on our data set. We also describe an interesting empirical relationship between the linear and nonlinear measures. The paper ends with Section 4 which contains a summary and discussion.

\section{Marginal Predictability and Seizure Anticipation}

\subsection{Definition of Marginal Predictability}

Consider the correlation integral (Grassberger and Procaccia, 1983) defined as

$$
C_{d}(y(i), y(j))=P\left(\left\|y^{(d)}(i)-y^{(d)}(j)\right\|<\varepsilon\right) .
$$

$\mathrm{P}(\cdot)$ is the probability of the argument, $x_{j}$ is the jth element of the time series being reconstructed, and $y^{(d)}(i)=\left(x_{i}, x_{i-1}, \ldots, x_{i-d+1}\right)$ is a $d$-dimensional vector reconstructed from data. The notation $\|\cdot\|$ means, in our calculations max norm of the argument which is computationally simple, i.e., $\left\|y^{(d)}(i)-y^{(d)}(j)\right\|<\varepsilon$ if $\max \left[\left|x_{i-k}-x_{j-k}\right|\right]<\varepsilon$ for $k=0,1, \ldots, d-1$.

The quantity $C_{d}$ is the probability that two vectors reconstructed from the time series in $d$-dimensions will be close to each other. In terms of the original time series, $C_{d}$ is a measure of the likelihood that two 
sequences of length $d$ taken from a time series will look similar.

Using the $C_{d}$ 's, we can define the predictability (Savit and Green, 1991) as

$$
S_{d}=\frac{C_{d+1}}{C_{d}},
$$

and using (1), we see that $S_{d}$ is just the conditional probability

$$
S_{d}=P\left(z_{d+1} \mid z_{d}, \ldots, z_{1}\right)
$$

where

$$
z_{k}=\left|x_{i+k-1}-x_{j+k-1}\right|<\varepsilon .
$$

In words, $S_{d}$ is the conditional probability that if two randomly chosen $d$-tuples from the time series have their first $d$-1 elements within $\varepsilon$ of each other, respectively, then the $d$ th elements will also be within $\varepsilon$.

$S_{d}$ can be used as a nonlinear statistic, but (Manuca and Savit, 1996) a more sensitive discriminator of nonlinear structure in time series is the ratio of $S_{d}$ 's, defined as

$$
R_{d}=\frac{S_{d}}{S_{d-1}}=\frac{C_{d+1} C_{d-1}}{C_{d}^{2}} .
$$

The marginal predictability (MP) is then defined as:

$$
\delta_{d} \equiv \frac{R_{d}-1}{R_{d}}
$$

$\delta_{d}$ is a measure of how much additional predictive information there is in the $(d+1)$ st lag of the time series, given that we have already used information in the intervening d lags. If $\delta_{d}$ is close to zero, then there is no additional predictive information, on average, for the current value of the time series in the value of the $(d+1)$ st lag. If $\delta_{d}$ is significantly different from zero, then $S_{d}>S_{d-1}$, and there is additional predictive information in the $(d+1)$ st lag. "Predictive information" here must be understood in the sense of nonlinear dynamics. See Savit and Green (1991) and Wu et al. (1993) for more details.

Our approach has been to compare $\delta_{d}$ for two different scalp electrodes as a function of time. Consider $Q_{d}(A, B ; t)=\delta_{d}(A ; t)-\delta_{d}(B ; t)$, where $A$ and $B$ are two electrodes and $t$ is time. Typically, A will be an electrode adjacent to the site of ictal onset and $B$ will be an electrode remote from the site of ictal onset.
In the case of mesiobasal temporal lobe epilepsy (MBTLE), we have generally found that the most consistent results obtain when both $A$ and $B$ are ipsilateral to the site of ictal onset. In this paper, we will report results for which $A$ is the temporal (adjacent) electrode (F7 or F8) and $B$ is the occipital (remote) electrode (O1 or $\mathrm{O} 2$ ), both electrodes being ipsilateral to the side of ictal onset. Thus we are interested in whether there is any difference in the marginal predictabilities of temporal and occipital electodes ipsilateral to the side of seizure onset between times far removed from a seizure and times close to a seizure.

\subsection{Marginal Predictability and Seizure Anticipation}

In our previous work $\mathrm{Li}$ et al. (2003a, 2003b) we showed, using several different tests, that $Q_{2}(A, B ; t)$ tended to decrease approximately 20-30 minutes prior to a seizure in patients with medically refractory MBTLE. In Li et al. (2003b) we used Wilcoxon's sum of positive rank test (SPR) on a sample size of 44 interictal epochs and 44 preictal epochs from a cohort of 14 patients. An interictal epoch is defined as one that is at least one hour away from any seizure. A preictal epoch is the hour immediately preceding a seizure. Using the (SPR), we showed that we had to reject the null hypothesis that $Q_{2}=0$ for interictal epochs, and for preictal epochs, up until about 30 minutes prior to a seizure. When the null hypothesis that $Q_{2}=0$ was rejected, we found that we had to accept the alternative hypothesis that $Q_{2}>0$. For times less than about 30 minutes prior to a seizure we could not reject the null hypothesis that $Q_{2}=0$. We also studied a variety of linear regressive models to try to reject the null hypothesis that $Q_{2}$ does not differ between preictal and interictal epochs. In these model tests, we used 61 interictal epochs of 20 minutes duration, and 33 preictal epochs consisting of the 20 minutes immediately prior to a seizure. Specifically, we tried to reject the null hypothesis that the value of $Q_{2}$ averaged over a 20 minute epoch, $\left\langle Q_{2}\right\rangle$, does not differ between preictal and interictal epochs. All of our results are consistent with rejection of this null hypothesis. In addition, we have used a range of linear regression models with this same data set to study the dependence of $\left\langle Q_{2}\right\rangle$ on behavior state. We have found that we cannot reject the null hypothesis that $\left\langle Q_{2}\right\rangle$ does not depend on behavior state. In sum, we have strong evidence, that $Q_{2}$ systematically decreases several tens 
of minutes prior to a seizure, and that $Q_{2}$ does not systematically depend on the behavior state of the patient. Details of these analyses can be found in $\mathrm{Li}$ et al. (2003b).

We parenthetically remark here that, not only do the aggregate results suggest that $Q_{2}$ decreases several tens of minutes prior to a seizure, but disaggregated results also support this view. In studies on individual patients, we have found that the value of $Q_{2}$ between occipital and temporal electrodes is a good discriminator of an impending seizure in 11 of 14 patients. Details of this analysis will be reported elsewhere $\mathrm{Li}$ et al. (2003c).

\section{Linear Measures, Nonlinear Structure and Seizure Anticipation}

\subsection{Nonlinear Structure in Epileptic Scalp Data: Surrogate Data Methods}

To test the hypthesis that thre is nonlinear structure in the scalp epileptic EEG data, we used the method of surrogate data (Schreiber and Schmitz, 2000) to construct linear surrogates for both the preictal and interictal epochs. In this approach, a Fourier transformation of the original data set is performed and the phases of the Fourier coefficients are randomized. An inverse Fourier transform of the phase randomized system is performed to produce a "linear surrogate data set". This linear surrogate data set has the same linear properties as the original data set, but has no specific nonlinear structure. A collection of such linear surrogate data sets, all with different realizations of the phase randomization are produced from the original data set, the metric of interest evaluated on the collection of linear surrogates, and compared with its value on the original data set. One simple measure that is used to determine whether the value of the metric on the original data is substantially different from its typical value on the surrogate data is the significance, $\Phi$, (Prichard and Theiler,
1994) defined by

$$
\Phi=\frac{\left|V_{o}-\bar{V}_{s}\right|}{\sigma_{s}(V)} .
$$

where $V_{o}$ is the value of the metric on the original data set, $\bar{V}_{s}$ is the mean of the values of $V$ computed on the surrogate data sets, and $\sigma_{s}$ is the standard deviation of those values. Values of $\Phi$ greater than about 3 are generally thought to indicate significant differences in the values of $V$ on the original and surrogate data sets.

We have performed this surrogate data analysis on 61 interictal and 33 preictal epochs of 20 minutes duration. Each 20 minute epoch was divided into 30, 40-second intervals. Each 40 second interval was considered as a separate time series for which 20 linear surrogate data sets were constructed. The results of our surrogate data analysis is presented in Table A, below. Here we list results for three different quantities, $\delta_{2}$ associated with the remote electrode, $\delta_{2}$ associated with the adjacent electrode and $Q_{2}$. For each of these quantities, we list the range of values of $\Phi$ for the preictal and interictal epochs, the mean of those values, and the range which includes $80 \%$ of the epochs whose values are distributed symmetrically about the median (i.e. after eliminating the smallest and largest $10 \%$ of the values). It is clear from this table that the behavior of our metrics is substantially different on the original data set as opposed to the linear surrogates. (Qualitatively, for example, we observe that the absolute values of $\delta_{2}$ are typically about an order of magnitude greater on the original data than on the linear surrogates.) In addition to the values of our metrics being much different on the original as opposed to the linear surrogate data sets, we find no evidence of qualitatively different behavior for our metrics on the linear surrogate data sets 20-30 minutes prior to a seizure, as we did for the original data sets. Thus, there is significant intrinsically nonlinear structure in scalp EEG recordings from subjects with epilepsy, that structure appears to carry

Table A. Significance of nonlinear quantities with respect to linear surrogates.

\begin{tabular}{lccccccc}
\hline & \multicolumn{3}{c}{$\Phi$, interictal epochs } & & \multicolumn{3}{c}{$\Phi$ preictal epochs } \\
\cline { 2 - 3 } \cline { 7 - 8 } Quantity & Range & Mean & Range, $80 \%$ & & Range & Mean & Range, 80\% \\
\hline$\delta_{2}$ (remote) & $1.2-833.1$ & 69.7 & $2.4-565.4$ & & $1.6-1245$ & 105.7 & $3.2-935.5$ \\
$\delta_{2}$ (adjacent) & $0.45-2355$ & 155.4 & $1.8-1037.7$ & & $1.1-3779$ & 173.9 & $2.8-3690.8$ \\
$Q_{2}$ & $1.7-214.7$ & 33.1 & $3.2-105.3$ & & $0.5-198.2$ & 31.9 & $1.2-143.7$
\end{tabular}


information about impending seizures, and our metrics are sensitive to that structure. ${ }^{1}$

\subsection{Linear Measures: Definitions and Methods}

There are, in principle, many linear measures associated with a time series. These can be thought of, for example, as the coefficients of an $\operatorname{AR}(n)$ fit to the data, with $n$ taken as large as is reasonable given the data. In this paper we will consider what are arguably the two simplest linear measures, namely, the standard deviation of the time series, and the coefficient of a standard linear AR(1) fit to the data. Specifically, for a time series $x(t)$ consisting of $N$ points, the standard deviation is determined by

$$
\begin{aligned}
\sigma^{2} & =\frac{1}{N} \sum_{t}[x(t)-\bar{x}]^{2}, \quad \text { with } \\
\bar{x} & =\frac{1}{N} \sum_{t} x(t) .
\end{aligned}
$$

The coefficient of a linear AR(1) fit, $a_{1}$, is determined by a best least-squares fit of the form

$$
x(t)=a_{0}+a_{1} x(t-1)+\eta
$$

where $\eta$ is the residual. Note that we do not claim that (9) is necessarily a good representation of the data. We use this only to define one of the two simplest linear measures whose behavior we are studying here.

In our case, we will consider $\sigma^{2}$ and $a_{1}$ computed for the twenty minute epochs of data derived from recordings of scalp electrodes that are adjacent to and remote from the site of ictal onset, as described in the paragraph following equation (6), above. We will use the same set of 94 20-minute epochs (61 interictal and 33 preictal) that were used in $\mathrm{Li}$ et al. (2003b). As in Li et al. (2003b), we take the data recorded from these electrodes at $200 \mathrm{~Hz}$, and construct from that data set a new time series consisting of every third data point. The technical reasons for this are described in detail in Li et al. (2003b). This decimated data set constitutes the time series $x(t)$ for which we compute $\sigma^{2}$ and $a_{1}$.

We will apply to $\sigma^{2}$ and $a_{1}$, the same set of statistical tests used in $\mathrm{Li}$, et al. (2003b)] to study the nonlinear measures $\delta_{2}$ and $Q_{2} . Q_{2}$ appears to be the nonlinear measure with the most power to discriminate between preictal and interictal epochs. Since $Q_{2}$ is the difference between the $\delta_{2}$ 's for the remote and adjacent electrodes, we will be particularly interested in the behavior of $\Delta \sigma^{2} \equiv \sigma^{2}$ (remote)- $\sigma^{2}$ (adjacent) and $\Delta a_{1} \equiv_{1}$ (remote)- $a_{1}$ (adjacent), although we will also study the dependence of the individual $\sigma^{2}$,s and $a_{1}$ 's.

As in our previous work, we construct six linear models to test for the dependence of $\sigma^{2}$ (remote), $\sigma^{2}$ (adjacent), $\Delta \sigma^{2}, a_{1}$ (remote), $a_{1}$ (adjacent) and $\Delta a_{1}$ on several variables that encompass (i) characterizations of the behavior state, (ii) the side on which the seizure focus is located, and (iii) whether the epoch in question is interictal or preictal as defined above. With regard to behavior states, we catalog each 20 minute epoch (61 interictal and 33 preictal) in our data set into a behavior state according to the following categories:

Awake, eyes open-AEO

Awake, eyes closed-AEC

Lightly drowsy-D1

Heavily drowsy-D2

Stage 2 NonREM sleep-S2

Stage 3 and 4 of NonREM sleep-S3/4

REM sleep-REM

From the set of 40 30-second intervals for a given epoch, a summary behavior score for that epoch was produced. If 32 or more of the 30 second intervals $(80 \%)$ of a given epoch were in the same behavior state, then that 20 minute epoch was deemed primarily in that behavior state e.g. AEO or D2. If 60-79\% of an epoch was spent in one state, then that epoch was considered as predominantly that state and indicated with the prefix P, thus PAEO or PD2. When less than $60 \%$ of an epoch was spent in just one state, then the epoch was considered a blend of 2 or more states, thus AEO/D2. Listed below are the numbers of various behavior states that comprise our basic data set of interictal and preictal epochs.
AEO-49
PAEO-3
D2-9
S2-17
Mixed states-16

No states were observed that were purely or predominantly D1, AEC, S3/4 or REM. However, the states D1 and AEC do contribute to some of the mixed states.

We will use this data to test for the dependence of $\sigma^{2}$ (remote), $\sigma^{2}$ (adjacent), $\Delta \sigma^{2}, a_{1}$ (remote), $a_{1}$ (adjacent) and $\Delta a_{1}$ for 20 minute epochs, on behavior state, on whether the epoch in question is preictal or interictal, 
and on whether the seizure focus is on the left or right side of the brain. The models become more complicated the more behavior state variables are included. For behavior states for which there are few observations it is therefore inadvisable to include additional variables. There are two approaches to this issue. The first is to agglomerate related behavior states (e.g. AEO and AEC) into one category. The second is to eliminate those states with few observations. We have done two separate analyses using each of these two approaches. In both cases, the number of behavior states against which we test for dependence in either case is four, AEO, D1, D2 and S2. For our first analysis we have used all 94 epochs of our data. This set we term "inclusive". In this approach we have categorized AEC observations with AEO observations. Note that the AEC observations only occur in mixed states. We have also performed the statistical tests on a subset of epochs in which we have eliminated those epochs and their associated behavior states for which there are only a few representatives. For this subset we have also removed all mixed states. All epochs used in this data set are either purely or predominately one behavior state. This data set we term "restricted". The total number of epochs that comprise the restricted data is 78 consisting of 54 interictal and 24 preictal epochs. As we shall see, our qualitative conclusions are the same for both data sets.

We introduce the following variables:

$X_{1}=1$ if the behavior state is AEO and $X_{1}=0$ otherwise

$X_{2}=1$ if the behavior state is D1, and $X_{2}=0$ otherwise

$X_{3}=1$ if the behavior state is $\mathrm{D} 2$, and $X_{3}=0$ otherwise

$X_{4}=1$ if the seizure focus is on the left side and $X_{4}=0$ if the seizure focus is on the right side

$X_{5}=1$ if the epoch is interictal and $X_{5}=0$ if the epoch is preictal

Note that since we use four categories of behavior state, the assignment $\left(X_{1}, X_{2}, X_{3}\right)=(0,0,0)$ uniquely corresponds to the behavior state $S 2$. An exception to the assignment of binary values to the $X_{1}, X_{2}$ and $X_{3}$ occurs in the characterization of mixed states for the inclusive data set. In this case the $X i$ take on values that reflect the fraction of the epoch associated with different behaviors. So, for example, a mixed state containing $25 \%$ of each of the four states, AEO, D1, D2 and S2 would be represented by the assignment $X_{1}=X_{2}=$ $X_{3}=0.25$.
In this paper we will present results for the six linear quantities $\sigma^{2}$ (remote), $\sigma^{2}$ (adjacent), $\Delta \sigma^{2}, a_{1}$ (remote), $a_{1}$ (adjacent) and $\Delta a_{1}$. Let $V$ stand for one of these quantities. We proceed in a standard way by constructing linear models, estimating coefficients in those models and computing $p$-values for the rejection of the null hypothesis that the values of the linear coefficient are zero. As in our previous work, we do not believe that the dependent variables, $V$, necessarily depend linearly on the variables, $X_{i}$. Rather, we adopt these linear tests because they are simplest way to look for some dependence of the variables, $V$ on the variables $X_{i}$. The following models and statistical tests were applied to each of these six linear quantities:

Test I: $V=a_{0}+a_{5} X_{5}$

Null hypothesis $a_{5}=0$, alternate hypothesis $a_{5} \neq 0$.

Test II: $V=a_{0}+a_{4} X_{4}$

Null hypothesis $a_{4}=0$, alternate hypothesis $a_{4} \neq 0$.

Test III: $V=a_{0}+a_{4} X_{4}+a_{5} X_{5}$

Null hypotheses $a_{4}=0$ or $a_{5}=0$ tested using partial F-tests.

Test IV: $V=a_{0}+a_{1} X_{1}+a_{2} X_{2}+a_{3} X_{3}$

Null hypothesis $a_{1}=a_{2}=a_{3}=0$, alternate hypothesis, at least one of the $a_{i}(i=1,2,3$, ) is non-zero.

Test $V: V=a_{0}+a_{5} X_{5}+a_{1} X_{1}+a_{2} X_{2}+a_{3} X_{3}$

Null hypothesis $a_{1}=a_{2}=a_{3}=0$, alternate hypothesis, at least one of the $a_{i}(i=1,2,3$, ) is non-zero.

Test VI: $V=a_{0}+\sum_{j=1}^{5} a_{j} X_{j}$

Null hypothesis $a_{1}=a_{2}=a_{3}=a_{4}=0$, alternate hypothesis, at least one of the $a_{i}(i=1.2 .3 .4)$ is non-zero.

Test $\mathrm{I}$ is a test for dependence on whether the epoch in question is preictal or interictal. Test II is a test for dependence on whether the seizure arises from the left or right side of the brain. Test III is an additional test for each of these dependencies after controlling for the other one. Test IV is a simple test for dependence on behavior state. Test $\mathrm{V}$ is a test for dependence on behavior state after controlling for whether the epoch is preictal or interictal, and test VI is a test for dependence on behavior state and/or whether the seizure arises from the left or right side, after controlling for whether the epoch is preictal or interictal.

\subsection{Linear Metrics: Results}

In Tables 1-6 in the Appendix we present the detailed results of our statistical tests. As is standard, we 
reject the null hypothesis (with $95 \%$ confidence) only if $p$-value $<0.05$. The results in these six tables support the following general conclusions:

1. There is no significant difference in our results between the inclusive and restricted data sets.

2. We cannot reject the null hypotheses that each of the linear measures have no dependence on whether the epoch is preictal or interictal, or whether the seizure arises from the right or left side of the brain.

3. For all the linear measures, with the exception of $\sigma^{2}$ (remote), we can reject the null hypothesis that there is no dependence on behavior state.

These results are in marked contrast to the results obtained for the nonlinear metrics $\left\langle Q_{2}\right\rangle$ and $\left\langle\delta_{2}\right\rangle$ (Li et al. (2003b)) for the same data set. In that case, we saw clear evidence of dependence of, most importantly, $\left\langle Q_{2}\right\rangle$ on whether the epoch in question was preictal or interictal, and no evidence of significant dependence on behavior state. These differences will be discussed further in the next section.

Given these results, it is interesting to look at one additional set of tests. We want to examine more directly, the relationship between some of the nonlinear measures and the linear measures. To this end, we consider the following statistical tests based on simple linear forms. As before, we do not necessarily believe that, if there is a functional relationship between the nonlinear and the linear variables, that that relationship is linear. We use these models only because they provide a simple test of the existence of a relationship (or lack thereof) between the nonlinear and linear quantities.

Test $A$ (applied separately to both the remote and adjacent electrodes): $\left\langle\delta_{2}\right\rangle=b_{0}+b_{1} \sigma^{2}$

Null hypothesis, $b_{1}=0$.

Test $B$ (applied separately to both the remote and adjacent electrodes): $\left\langle\delta_{2}\right\rangle=b_{0}+b_{2} a_{1}$

Null hypothesis, $\mathrm{b}_{2}=0$

Test $C:\left\langle Q_{2}\right\rangle=c_{0}+c_{1}\left(\Delta \sigma^{2}\right)$

Null hypothesis $\mathrm{c}_{1}=0$

Test D: $\left\langle Q_{2}\right\rangle=c_{0}+c_{2}\left(\Delta a_{1}\right)$

Null hypothesis $\mathrm{c}_{2}=0$

Test $E:\left\langle Q_{2}\right\rangle=c_{0}+c_{1}\left(\Delta \sigma^{2}\right)+c_{2}\left(\Delta a_{1}\right)$

Null hypotheses, $c_{1}=0$ or $c_{2}=0$, tested using partial F-tests.

The results of these tests for both the inclusive and restricted data sets appear in Table 7 of the Appendix. It is clear from this table that there is some relationship between the values of $\left\langle\delta_{2}\right\rangle$ and $\sigma^{2}$ for the electrode adjacent to site of ictal onset. This relationship is also, evidently, reflected in the relationship between $\left\langle Q_{2}\right\rangle$ and $\Delta \sigma^{2}$. On the other hand, this linear test is not able to reject the null hypothesis of no relation between $\left\langle\delta_{2}\right\rangle$ and $\sigma^{2}$ for the remote electrode. Finally, in one of our data sets, there appears to be a relationship between $\left\langle\delta_{2}\right\rangle$ and $a_{1}$ for the adjacent electrode. However, since we only reject the null hypothesis for the inclusive data set, and since we accept the null hypothesis in test $\mathrm{E}$ that $c_{2}=0$ for both data sets, there is reason to be skeptical of this relationship. We should, however, take seriously the apparent relationship between $\left\langle\delta_{2}\right\rangle$ and $\sigma^{2}$ for the adjacent electrode, and the consequent relationship between $\left\langle Q_{2}\right\rangle$ and $\Delta \sigma^{2}$. Since $\left\langle Q_{2}\right\rangle$ is the nonlinear measure of primary interest to us, we will focus on that quantity. To help clarify the possible relationship between $\left\langle Q_{2}\right\rangle$ and $\Delta \sigma^{2}$, it is useful to look at a scatter plot, for these two variables. This is presented in Fig. 1. Note that most of the points are clustered, but there is one far outlier. This epoch was associated with more that the usual amount of muscle artifact. We can redo Test $\mathrm{C}$ on a reduced data set that eliminates this outlier. The result is presented in the last row of Table 7. After eliminating this outlying epoch, we can no longer reject the null hypothesis of no relation between $\left\langle Q_{2}\right\rangle$ vs. $\Delta \sigma^{2}$. It is also important to note that even with this outlier eliminated, we still find a significant relationship between $\left\langle Q_{2}\right\rangle$ and whether the epoch in question is preictal or interictal. In particular, if we let $V=\left\langle Q_{2}\right\rangle$, then in Test I above, we reject the null hypothesis with a $p$-value of 0.038 .

Thus, the apparent relationship between $\left\langle Q_{2}\right\rangle$ vs. $\Delta \sigma^{2}$ can be eliminated by removing a single outlying point, and removal of that epoch does not affect our conclusions about the relationship between $\left\langle Q_{2}\right\rangle$ and whether the epoch is preictal or interictal. That said, it is important to note that while we find no strong evidence for a linear relationship between $\left\langle Q_{2}\right\rangle$ and $\Delta \sigma^{2}$, it is possible that there is a more subtle relationship. Indeed, an inspection of Fig. 1 shows that the range of values of $\left\langle Q_{2}\right\rangle$ is larger when $\Delta \sigma^{2}$ is smaller, even thought there is no systematic linear relation between them.

\section{Summary and Discussion}

In Li et al. (2003a), we introduced a new nonlinear measure based on the analysis of scalp EEG recordings, and demonstrated a relationship between values of that measure and whether an epoch was in close temporal 


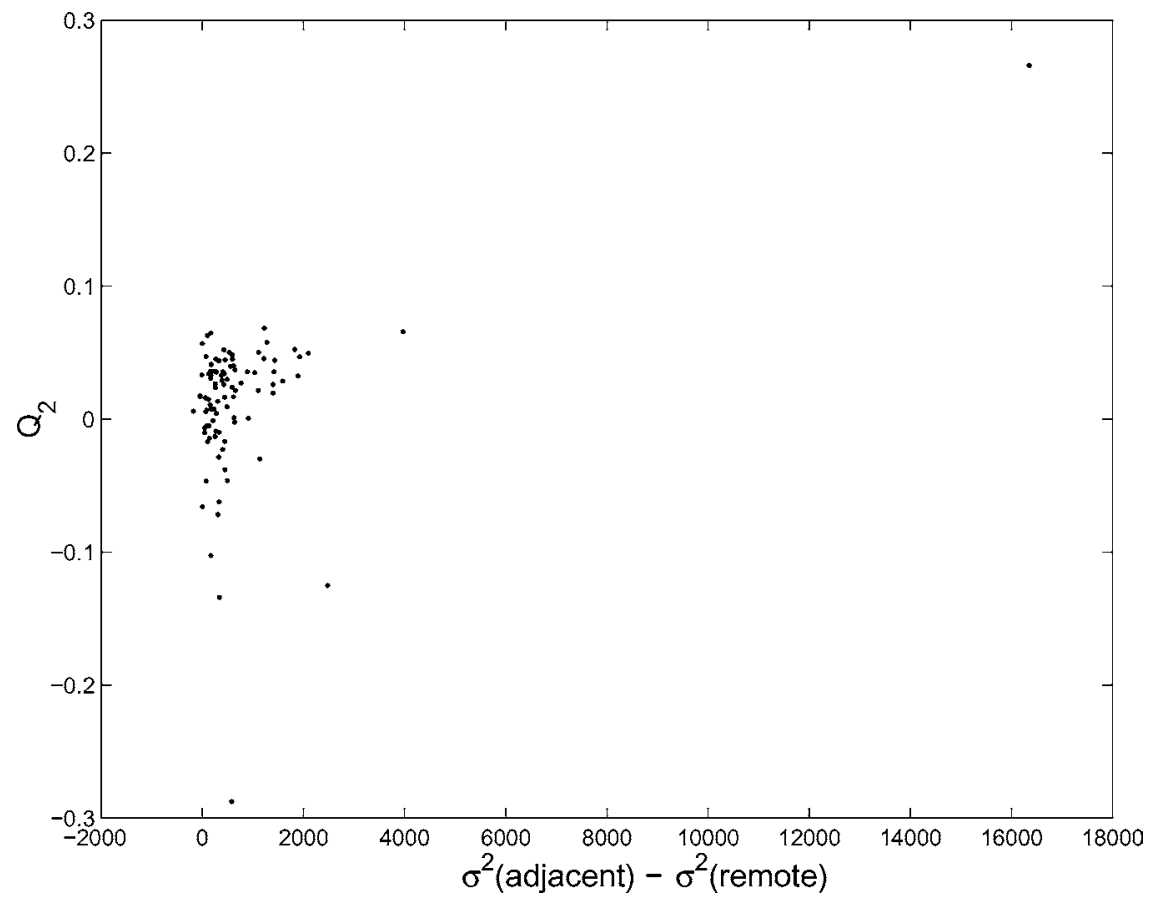

Figure 1. $\left\langle Q_{2}\right\rangle$ vs. $\Delta \sigma^{2}$ including all 94 epochs of the inclusive data set.

proximity to a seizure in patients with mesiobasal temporal lobe epilepsy. In Li et al. (2003b), we extended our analysis to include a much larger data sample. We showed there additional support for the power of $\left\langle Q_{2}\right\rangle$ to discriminate between preictal and interictal epochs, and we showed also that $\left\langle Q_{2}\right\rangle$ was relatively insensitive to the behavior state of the patient.

In this paper we have studied the behavior of our metrics on linear surrogates of our data, and we have also studied the question of whether simple linear measures can reproduce the discriminative power of $\left\langle Q_{2}\right\rangle$, an intrinsically nonlinear measure, on scalp EEG from epileptic subjects. We have shown that our metrics behave very differently on linear surrogates and that this linearization appears to remove information that signals an impending seizure. Returning to the original scalp EEG data set, we have shown that there is little relation between the simplest linear measures, namely the standard deviation and the first linear auto-regressive coefficient, and our nonlinear measures on the data. Notably, the linear measures are relatively sensitive to the behavior state of the subject but are insensitive to whether the epoch preictal or interictal. On the other hand, the measure $\left\langle Q_{2}\right\rangle$ is sensitive to whether the epoch is preictal or interictal, but is insensitive to the be- havior state of the subject. The only observed relationship between the linear and nonlinear measures is evidently attributable to the effect of a far outlying epoch.

It is important to study the relationship between linear and nonlinear measures for several reasons. First, $\left\langle\delta_{2}\right\rangle$ and $\left\langle Q_{2}\right\rangle$ on the one hand, and $\sigma^{2}$ and $\Delta \sigma^{2}$ on the other are, a priori, sensitive to very different features of the data. If there were a nontrivial relationship between them it might have implications for the underlying dynamics of ictal genesis. In this regard, it is important to mention the relationship between the values of $\left\langle Q_{2}\right\rangle$ and $\Delta \sigma^{2}$ that we pointed out in the last section, namely, that the spread in values of $\left\langle Q_{2}\right\rangle$ is larger when $\Delta \sigma^{2}$ is smaller, even thought there is no systematic linear relation between them. The dynamical implications of this observation are unclear, but a deeper investigation of it is clearly warranted. Second, since linear measures are typically easier to compute than nonlinear ones, a demonstration that the linear measures are as sensitive as nonlinear measures to the preictal or interictal nature of the epoch would have had practical implications, possibly resulting in a simpler strategy for seizure anticipation. This, however, appears not to be the case. Absent a systematic relation between the expected values of linear and nonlinear measures, it is 
hard to see how linear metrics could easily supplant the use of nonlinear metrics in a protocol such as the one we consider. Finally, and to avoid confusion, we make the following technical remark. A paper by Martinerie et al. (1998) has been criticized by McSharry et al. (2003). These latter authors demonstrated that the behavior of a nonlinear metric observed in Martinerie et al. (1998) could be explained by the behavior of the standard deviation of the time series that those authors studied. The technical origin of this observation is, essentially, that Martinerie et al. did not properly renormalize their computation of the correlation integral to remove the large effect of an increased spread of the data (and the consequent reduction in the value of the correlation integral) that is associated with an increased standard deviation. Our approach is immune from this criticism since we renormalized all the data in each 30 second window, 40 of which comprise one of our 20 minute epochs.

Although the analyses of our data is strongly suggestive that $\left\langle Q_{2}\right\rangle$ could be the basis for a robust seizure anticipation protocol, the most important test of this possibility will be in studies on disaggregated data. In
Li et al. (2003b) we noted that in 11 of 14 patients with temporal lobe epilepsy $\left\langle Q_{2}\right\rangle$ was systematically lower in preictal than in interictal epochs. There is reason to be optimistic that, at least in a significant subset of patients with localization-related epilepsy, a protocol similar to the one suggested here will be useful. Not only do we have promising preliminary results, but our approach also admits of the possibility of tailoring the protocols to different patients. There are a number of control parameters in the algorithms (e.g. $\varepsilon, \tau$, window length, reconstruction dimension) that can be varied to optimize the reliability of the protocol for different patients. Because of the heterogeneity of even a relatively well-defined syndrome such as mesiobasal temporal lobe epilepsy, it is not unreasonable that different parameter settings in a seizure anticipation protocol may be appropriate for different patients. Our results are, indeed, very promising, but much more work needs to be done. A much larger database needs to be studied and out of sample tests need to be performed. We are currently pursuing these studies.

\section{Appendix}

Table 1. Summary of dependence tests for $\left\langle\sigma^{2}\right\rangle$, focal electrode.

\begin{tabular}{lccccc}
\hline & $\left\langle\sigma^{2}\right\rangle$ inclusive data set Adjacent (temporal) electrode & & $\left\langle\sigma^{2}\right\rangle$ restricted data set Adjacent (temporal) electrode \\
\cline { 2 - 3 } & Null hypothesis & $p$-value & & Null hypothesis & $p$-value \\
\hline Test I & Accept & 0.80 & Accept & 0.55 \\
Test II & Accept & 0.82 & Accept & 0.84 \\
Test III & (Accept, accept $)$ & $0.83,0.81$ for $\left(a_{4}, a_{5}\right)=0$ & & (Accept, accept) & $0.797,0.537$ for $\left(a_{4}, a_{5}\right)=0$ \\
Test IV & Reject & 0.02 & & Reject & 0.034 \\
Test V & Reject & 0.012 & Reject & 0.012 \\
Test VI & Reject & 0.027 & & Reject & 0.083 \\
\hline
\end{tabular}

Table 2. Summary of dependence tests for $\left\langle\sigma^{2}\right\rangle$, remote electrode.

\begin{tabular}{|c|c|c|c|c|}
\hline & \multicolumn{2}{|c|}{$\left\langle\sigma^{2}\right\rangle$ inclusive data set Remote (occipital) electrode } & \multicolumn{2}{|c|}{$\left\langle\sigma^{2}\right\rangle$ restricted data set Remote (occipital) electrode } \\
\hline & Null hypothesis & $p$-value & Null hypothesis & $p$-value \\
\hline Test I & Accept & 0.14 & Accept & 0.60 \\
\hline Test II & Accept & 0.09 & Accept & 0.13 \\
\hline Test III & (Accept, accept) & $0.08,0.12$ for $\left(a_{4}, a_{5}\right)=0$ & (Accept, accept) & $0.17,0.08$ for $\left(a_{4}, a_{5}\right)=0$ \\
\hline Test IV & Accept & 0.94 & Accept & 0.80 \\
\hline Test V & Accept & 0.83 & Accept & 0.85 \\
\hline Test VI & Accept & 0.40 & Accept & 0.30 \\
\hline
\end{tabular}


Table 3. Summary of dependence tests for $\left\langle\Delta \sigma^{2}\right\rangle$, remote electrode.

\begin{tabular}{lccccc}
\hline & \multicolumn{2}{c}{$\left\langle\Delta \sigma^{2}\right\rangle$ inclusive data set } & & \multicolumn{2}{c}{$\left\langle\Delta \sigma^{2}\right\rangle$ restricted data set } \\
\cline { 2 - 3 } & Null hypothesis & $p$-value & & Null hypothesis & $p$-value \\
\hline Test I & Accept & 0.38 & & Accept & 0.42 \\
Test II & Accept & 0.10 & Accept & 0.14 \\
Test III & (Accept, accept $)$ & $0.09,0.35$ for $\left(a_{4}, a_{5}\right)=0$ & & (Accept, accept $)$ & $0.16,0.50$ for $\left(a_{4}, a_{5}\right)=0$ \\
Test IV & Reject & 0.0095 & & Reject & 0.02 \\
Test V & Reject & 0.014 & & Reject & 0.027 \\
Test VI & Reject & 0.0089 & & Reject & 0.035 \\
\hline
\end{tabular}

Table 4. Summary of dependence tests for $\langle\mathrm{AR} 1\rangle$, adjacent electrode.

\begin{tabular}{|c|c|c|c|c|}
\hline & \multicolumn{2}{|c|}{$\langle\mathrm{AR} 1\rangle$ inclusive data set Adjacent (temporal) electrode } & \multicolumn{2}{|c|}{$\langle A R 1\rangle$ restricted data set Adjacent (temporal) electrode } \\
\hline & Null hypothesis & $p$-value & Null hypothesis & $p$-value \\
\hline Test I & Accept & 0.61 & Accept & 0.77 \\
\hline Test II & Accept & 0.95 & Accept & 0.22 \\
\hline Test III & (Accept, accept) & $0.94,0.61$ for $\left(a_{4}, a_{5}\right)=0$ & (Accept, accept) & $0.21,0.69$ for $\left(a_{4}, a_{5}\right)=0$ \\
\hline Test IV & Reject & 0.011 & Reject & 0.0385 \\
\hline Test V & Reject & 0.009 & Reject & 0.033 \\
\hline Test VI & Reject & 0.018 & Accept & 0.052 \\
\hline
\end{tabular}

Table 5. Summary of dependence tests for $\langle\mathrm{AR} 1\rangle$, remote electrode.

\begin{tabular}{|c|c|c|c|c|}
\hline & \multicolumn{2}{|c|}{$\langle A R 1\rangle$ inclusive data set Remote (occipital) electrode } & \multicolumn{2}{|c|}{$\langle\mathrm{AR} 1\rangle$ restricted data set Remote (occipital) electrode } \\
\hline & Null hypothesis & $p$-value & Null hypothesis & $p$-value \\
\hline Test I & Accept & 0.27 & Accept & 0.70 \\
\hline Test II & Accept & 0.22 & Accept & 0.60 \\
\hline Test III & (Accept, accept) & 0.230 .28 for $\left(a_{4}, a_{5}\right)=0$ & (Accept, accept) & $0.58,0.67$ for $\left(a_{4}, a_{5}\right)=0$ \\
\hline Test IV & Reject & 0.005 & Reject & 0.005 \\
\hline Test V & Reject & 0.008 & Reject & 0.006 \\
\hline Test VI & Reject & 0.001 & Reject & 0.01 \\
\hline
\end{tabular}

Table 6. Summary of dependence tests for $\langle\Delta \mathrm{AR} 1\rangle$, remote electrode.

\begin{tabular}{|c|c|c|c|c|}
\hline & \multicolumn{2}{|c|}{$\langle\Delta \mathrm{AR} 1\rangle$ inclusive data set } & \multicolumn{2}{|c|}{$\langle\Delta \mathrm{AR} 1\rangle$ restricted data set } \\
\hline & Null hypothesis & $p$-value & Null hypothesis & $p$-value \\
\hline Test I & Accept & 0.34 & Accept & 0.61 \\
\hline Test II & Accept & 0.10 & Accept & 0.32 \\
\hline Test III & (Accept, accept) & 0.190 .36 for $\left(a_{4}, a_{5}\right)=0$ & (Accept, accept) & $0.29,0.55$ for $\left(a_{4}, a_{5}\right)=0$ \\
\hline Test IV & Reject & 0.02 & Reject & 0.043 \\
\hline Test V & Reject & 0.035 & Reject & 0.05 \\
\hline Test VI & Reject & 0.035 & Accept & 0.06 \\
\hline
\end{tabular}


Table 7. Linear tests of the existence of relationships between nonlinear and linear measures.

\begin{tabular}{|c|c|c|c|c|}
\hline & \multicolumn{2}{|c|}{ Inclusive data set } & \multicolumn{2}{|c|}{ Restricted data set } \\
\hline & Null hypothesis & $p$-value & Null hypothesis & $p$-value \\
\hline Test A (adjacent) & Reject & 0.0017 & Reject & 0.0003 \\
\hline Test A (remote) & Accept & 0.313 & Accept & 0.254 \\
\hline Test B (adjacent) & Reject & 0.0156 & Accept & 0.500 \\
\hline Test C & Reject & 0.0001 & Reject & 0.0001 \\
\hline Test D & Accept & 0.9393 & Accept & 0.905 \\
\hline Test E & (Reject, accept) & $(0.0001,0.2741)$ for $\left(c_{1}, c_{2}\right)$ & (Reject, accept) & $(0.0001,0.860)$ for $\left(c_{1}, c_{2}\right)$ \\
\hline Test C (outlier removed-93 epochs) & Accept & 0.204 & & \\
\hline
\end{tabular}

\section{Acknowledgments}

This work was supported in part by the National Institutes of Health under grant no. RO1 NS36803-01A1.

\section{Note}

1. Although nonlinear effects dominate the linear structure in the original data set, as far as our metrics are concerned, there is still interesting linear structure in these data. We have performed an additional analysis of our data by taking the linear surrogates and treating them as "original" data sets. For a given linear surrogate, we constructed a set of IID surrogates that removes the linear structure, turning the time series into a random (IID) series. Using our metrics, we have found some interesting structure in the linear surrogates that are absent in the IID surrogates. A detailed discussion of this finding is beyond the scope of this paper, but will be presented elsewhere.

\section{References}

Grassberger P, Procaccia I (1983) Measuring the strangeness of strange attractors. Physica D 9: 189-208.

Jerger K, Netoff T, Francis J, Sauer T, Pecora L, Weinstein S, Schiff S (2001) Early seizure detection. J. Clin. Neurophysiology 18: 259.

Li D, Zhou W, Drury I, Savit R (2003a) Nonlinear, non-invasive method for seizure anticipation in focal epilepsy. Math. Bio. 186: 63-77.
Li D, Zhou W, Drury I, Savit R (2003b) Seizure anticipation, behavior states and marginal predictability in temporal lobe epilepsy. submitted to Journal of Neuroscience.

Li D, Zhou W, Drury I, Savit R (2003c) Seizure anticipation and marginal predictability in individual patients with temporal lobe epilepsy. in preparation.

McSharry P, Smith L, Tarassenko L (2003) Prediction of epileptic seizures: Are nonlinear methods relevant? Nat. Med. 4: 11731176.

Manuca R, Savit R (1996) Stationarity and nonstationarity in time series analysis. Physica D, 99: 134

Martinerie J, Adam C, Le Van Quyen M, Baulac M, Clemenceau S, Renault B, Varela F (1998) Epileptic seizures can be anticipated by non-linear analysis. Nature Medicine 4: 1173-1176.

Paulus M (1993) In Nonlinear Dynamical Analysis of the EEG, B. Jansen, M. Brandt eds. World Scientific, Singapore, p. 100.

Pritchard W, Duke D (1992) Measuring chaos in the brain: A tutorial review of nonlinear dynamical EEG analysis. Int. J. Neurosci. 67: 31.

Prichard D, Theiler J (1994) Generating surrogate data for time series with several simultaneously measured variables. Phys. Rev. Lett. 73: 951-954.

Rapp P (1992) Chaos in the neurosciences: Cautionary tales from the frontier. Biologist 40: 89 .

Savit R, Green M (1991) Time series and dependent variables. Physica D 50: 95 (1991).

Schreiber T, Schmitz A (2000) Surrogate time series. Physics D 142: 346-382.

Theiler J (1995) On the evidence for low-dimensional chaos in an epileptic electroencephalogram. Phys. Lett A 196: 335.

Wu K, Brock W, Savit R (1993) Statistical tests for deterministic effects in complex time series. Physica D 69: 172. 